\title{
Inhibition of hatching of mouse blastocysts in vitro by various prostaglandin antagonists
}

\author{
J. F. Baskar*, D. F. Torchiana, J. D. Biggers, E. J. Corey†, \\ N. H. Andersen $\neq$ and N. Subramanian $\ddagger$
}

Department of Physiology and Laboratory of Human Reproduction and Reproductive Biology, Harvard Medical School, Boston, Massachusetts 02115;

$\uparrow$ Department of Chemistry, Harvard University, Cambridge, Massachusetts 02138, and

$\ddagger$ Department of Chemistry, University of Washington, Seattle, Washington 98195, U.S.A.

\begin{abstract}
Summary. Hatching of mouse blastocysts in vitro is inhibited by 18,18,20-trimethyl PGE-2, 17,17-dimethyl PGE-2, 8,12-epiPGE-2 and $N$-dimethylamide PGF-2 $\alpha$, whose activity is between that of 7-oxa-13-prostynoic acid and meclofenamic acid.
\end{abstract}

\section{Introduction}

The hatching of mouse blastocysts in vitro is inhibited by several classes of prostaglandin antagonists (Biggers, Leonov, Baskar \& Fried, 1978). The two most active compounds studied were 7-oxa-13-prostynoic acid and meclofenamic acid. The mode of action of prostaglandin antagonists may be complex, involving single or multiple sites of action. There is evidence that the fenamic acids competitively bind to the cyclo-oxygenase component of the prostaglandin synthetase system and thus inhibit the synthesis of all components of the arachidonic acid cascade (Cushman \& Cheung, 1976). 7-Oxa-13-prostynoic acid may inhibit prostaglandin synthesis (McDonald-Gibson,. Flack \& Ramwell, 1973), and also decrease prostaglandin binding (Bennett, 1974). Nevertheless, the compound also has non-specific effects since it affects the catalytic sub-unit of adenylate cyclase (Hynie et al., 1975).

Attempts have been made to synthesize more specific antagonists of prostaglandins (PG) F-2 $\alpha$ and E-2. These efforts can be justified because the spatial conformations of PGF-2 $\alpha$ and PGE-2 are very distinct (Langs, Erman \& DeTitta, 1977), and they have little affinity for each other's receptors (Kimball, Lauderdale, Nelson \& Jackson, 1976). Possible antagonists of PGE-2 are 18,18,20-trimethyl PGE-2 and 17,17-dimethyl PGE-2, synthesized by Andersen et al. (1979), and 8,12-epiPGE-2, synthesized by Andersen \& Imamoto (1976) and Andersen, Imamoto \& Picker (1977). A possible inhibitor of PGF-2 $\alpha$ is $N$-dimethylamide PGF-2 $\alpha$, synthesized by Maddox, Ramwell, Shiner \& Corey (1978). The action of all four compounds on the hatching of mouse blastocysts in vitro has been examined.

\section{Materials and Methods}

Animals and collection and culture of embryos

The random-bred albino Swiss mice, CF1 from Charles River Breeding Laboratory, Wilmington, Massachusetts, were 6-8 weeks old when used. Superovulation was induced by

- Present address: The Cancer Research Center, University of North Carolina at Chapel Hill, Clinical Science Building 229H, Chapel Hill, North Carolina 27514, U.S.A. 
injection of 5 i.u. PMSG (Sigma, St Louis, Missouri) followed $48 \mathrm{~h}$ later by 5 i.u. hCG (Sigma). Each female was placed with a male overnight and insemination was verified the next morning by the observation of a vaginal plug. Blastocysts were flushed from the uterus 3.5 days later with the medium used for culture. The blastocysts were pooled from several animals for each assay.

The blastocysts were cultured in a protein-free modification of the medium described by Goldstein, Spindle \& Pedersen (1975), using the techniques described by Biggers et al. (1978). After incubation for $24 \mathrm{~h}$ the embryos in each culture vessel were scored for the number of blastocysts which had hatched.

\section{Inhibitors}

17,17-Dimethyl PGE-2, 18,18,20-trimethyl PGE-2 and 8,12-epiPGE-2. The compounds (mol. wt 380, 394 and 350 respectively) were supplied in 50\% ethanol. These solutions were diluted with the culture medium described below to give a $1 \mathrm{~mm}$ stock solution which was distributed in $100 \mu \mathrm{l}$ aliquots to screw-capped vials and stored at $-20^{\circ} \mathrm{C}$.

$N$-Dimethylamide PGF-2a A $10 \mathrm{~mm}$ solution of the substance (mol. wt 381) was prepared in $50 \%$ ethanol and diluted with culture medium to give a $1 \mathrm{mM}$ stock solution which was placed in $100 \mu \mathrm{l}$ aliquots in screw-capped vials and stored at $-20^{\circ} \mathrm{C}$.

\section{Experimental design and statistical analysis}

Blastocysts were incubated in the presence of an inhibitor. The doses used in each assay were adjusted from assay to assay in the light of previous results. In all assays a control group, not exposed to inhibitor, was included to determine the natural rate of hatching. The data from each assay were analysed by fitting a probit response-log dose line, using the procedures described by Biggers et al. (1978) to adjust for the natural rate of hatching. A $\chi^{2}$ test of significance was used to test the goodness of fit of each regression line.

\section{Results}

The results of the 7 assays with 18,18,20-trimethyl PGE-2 are shown in Table 1 . An estimate of the slope $(\hat{\beta})$ and its variance and an estimate of the $\log \operatorname{ID}_{50}(\hat{\mu})$ and its variance were calculated for each assay. The 7 estimates of the slope were tested for homogeneity using a $\chi^{2}$ test of significance (Emmens, 1948), and it was found that they formed a homogeneous group. The 7 estimates of the $\mathrm{ID}_{30}$ were similarly shown to form a homogeneous group. The results of the $\chi^{2}$ tests and the weighted means are summarized in Table 2.

Table 1. The number of blastocysts hatching from the number incubated in various concentrations of 18,18,20-trimethyl PGE-2

\begin{tabular}{ccccccc}
\hline $\begin{array}{c}\text { Dose } \\
(\mu \mathrm{M})\end{array}$ & I & II & III & IV & V & VI \\
\hline 0 (control) & $14 / 27$ & $11 / 21$ & $10 / 20$ & $8 / 22$ & $16 / 30$ & $12 / 22$ \\
$0 \cdot 5$ & $8 / 19$ & - & - & - & - & - \\
1 & $7 / 17$ & - & - & - & - & - \\
2 & $6 / 14$ & $9 / 15$ & $8 / 16$ & $9 / 21$ & $8 / 18$ & $11 / 21$ \\
4 & $3 / 17$ & $4 / 14$ & $5 / 15$ & $7 / 20$ & $5 / 20$ & $4 / 20$ \\
8 & $2 / 20$ & $1 / 15$ & $2 / 16$ & $1 / 20$ & $1 / 20$ & $0 / 21$ \\
16 & $0 / 20$ & $0 / 16$ & $2 / 20$ & $0 / 20$ & - & $0 / 20$ \\
\hline
\end{tabular}


The inhibitors 8,12-epiPGE-2 and $N$-dimethylamide PGF- $2 \alpha$ were tested in 5 and 8 assays respectively. The two sets of dose-response lines also formed homogeneous groups (Table 2). Although the confidence limits for the $\mathrm{ID}_{50}$ were wide for 8,12-epiPGE-2 and $N$-dimethylamide PGF- $2 \alpha$ the results suggest that $18,18,20$-trimethyl PGE-2 is the most active of the three compounds.

Table 2. Estimates of the parameters of the regression of the probit of proportion of blastocysts hatching on the log dose of 18,18,20-trimethyl PGE-2, 17,17-dimethyl PGE-2, 8,12-epiPGE-2 and $N$-dimethylamide PGF-2 $\alpha$, after adjustment for the control rate of hatching

\begin{tabular}{|c|c|c|c|}
\hline Parameter & $\begin{array}{l}\text { 18,18,20-Trimethyl } \\
\text { PGE-2 }\end{array}$ & 8,12-Epi-PGE-2 & $\begin{array}{l}N \text {-Dimethylamide } \\
\text { PGF-2 } \alpha\end{array}$ \\
\hline \multicolumn{4}{|l|}{$\log I_{50}$} \\
\hline Homogeneity & $x_{\{3\}}^{2}=0.034$ & $\chi_{[4]}^{2}=0.296$ & $\chi_{[7]}^{2}=0.054$ \\
\hline$P$ & 1.00 & 0.99 & 1.00 \\
\hline Weighted mean & 0.603 & 1.214 & 1.337 \\
\hline Standard error & 0.293 & 0.294 & 0.421 \\
\hline $\mathrm{ID}_{s 0}(\mu \mathrm{M})$ & 4.01 & $16 \cdot 4$ & 21.7 \\
\hline Confidence limits $(P=0.05)$ & $1.07-15 \cdot 1$ & $4 \cdot 34-61 \cdot 7$ & $3 \cdot 24-145$ \\
\hline \multicolumn{4}{|l|}{ Regression coefficient $\left(\hat{\beta}_{w}\right)$} \\
\hline Homogeneity & $\chi_{[s]}^{2}=3 \cdot 13$ & $\chi_{[4]}^{2}=0.219$ & $\chi_{171}^{2}=5.291$ \\
\hline$P$ & 0.68 & 0.04 & 0.62 \\
\hline Weighted mean & $3 \cdot 231$ & $3 \cdot 146$ & $2 \cdot 110$ \\
\hline Standard error & 0.688 & 0.401 & 0.324 \\
\hline $\begin{array}{l}\text { Standard deviation of the tolerance } \\
\text { distributon }\left(\hat{\sigma}_{2}=1 / \hat{\beta}_{w}\right)\end{array}$ & 0.310 & 0.318 & 0.474 \\
\hline $\begin{array}{l}\text { Coefficient of variation }(\eta) \text { of the } \\
\text { tolerance doses }(x)\end{array}$ & 0.317 & 0.326 & 0.502 \\
\hline
\end{tabular}

The data from 4 assays with 17,17-dimethyl PGE-2 were insufficient to estimate the weighted mean $\mathrm{ID}_{50}$ but inspection of the individual dose-response lines showed that the $\mathrm{ID}_{50}$ was $>20 \mu \mathrm{M}$.

The estimated tolerance distributions of doses of 18,18,20-trimethyl PGE-2 and $\mathrm{N}$ dimethylamide PGF-2 $\alpha$ are shown in Text-fig. 1. They were computed by allotting the values of $\hat{\mu}_{w}$ and $\hat{\sigma}_{w}=1 / \hat{\beta}_{w}$ to the parameters of the log normal distribution (Biggers et al., 1978).

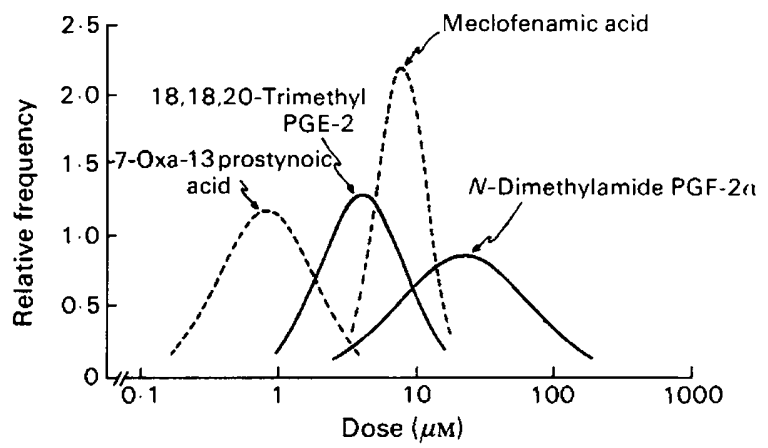

Text-fig. 1. The estimated tolerance distributions of the doses of 18,18,20-trimethyl PGE-2 and $N$-dimethylamide PGF-2 $\alpha$ which just prevent hatching of mouse blastocysts in vitro. The distributions are compared with the estimated tolerance distributions for 7-oxa-13-prostynoic acid and meclofenamic acid described by Biggers $e t$ al. (1978). 


\section{Discussion}

The results presented in this paper extend the list of compounds than can inhibit the hatching of mouse blastocysts in vitro. The most active is 18,18,20-trimethyl PGE-2, a possible inhibitor of PGE-2. Its activity appears to be less than that of 7-oxa-13-prostynoic acid and greater than that of meclofenamic acid, whose activities have been described elsewhere (Text-fig. 1). All three compounds reduce the fertility of mice when instilled into the uterus at the expected time of implantation (Biggers, Baskar \& Torchiana, 1981).

Biggers et al. (1978) argued that fluid accumulation and hatching of the mouse blastocyst were dependent on adequate supplies of PGE-2. The high activity of 18,18,20-trimethyl PGE-2 in inhibiting hatching would be fully consistent with this view if the analogue were a truly specific inhibitor of PGE-2. $N$-Dimethylamide PGF-2 $\alpha$, a possible antagonist of PGF-2 $\alpha$, is also active, albeit in relatively high amounts. This result must be considered in the light of the presence of PGF-2 $\alpha$ and PGE-2 in the rabbit blastocyst (Dickmann \& Spilman, 1975; Dey, Chein, Cox \& Crist, 1980), and the demonstration that PGE-2 and PGF-2 $\alpha$ can be synthesized by the rabbit blastocyst from endogenous arachidonic acid (C. Rice, J. D. Biggers \& L. Levine, unpublished data). Therefore, both PGE- 2 and PGF-2 $\alpha$ may be involved in the functions of the mammalian blastocyst. If 18,18,20-trimethyl PGE-2 and $N$-dimethylamide PGF- $2 \alpha$ are specific inhibitors of PGE-2 and PGF-2 $\alpha$ respectively, the results presented in this paper would show that both classes of prostaglandin are involved in fluid accumulation and hatching of the blastocyst in vitro. This conclusion contrasts with the suggestion that only PGE-2 plays a critical role in hatching of the mouse blastocyst. However, the specificities of 18,18,20-trimethyl PGE-2 and $N$-dimethylamide PGF- $2 \alpha$ are still unproven, and therefore the role of specific prostaglandins in fluid formation in the blastocyst remains unclear.

The work reported in this paper was supported in part by grants from the Rockefeller Foundation (RF-65040), the National Institute of Child Health and Human Development (HD-06916), and the National Science Foundation.

\section{References}

Andersen, N.H. \& Imamoto, S. (1976) An alternative prostaglandin analog synthesis strategy. Methylation sequence for bis-unsaturated prostaglandins. Synthetic Commun. 6, 33-38.

Andersen, N.H., Imamoto, S. \& Picker, D.H. (1977) Synthesis of diastereomeric bis-unsaturated prostaglandins. Prostaglandins 14, 61-101.

Andersen, N.H., Imamoto, S., Subramanian, B., De, B., Eggerman, T., Harker, L.A. \& Robertson, R.P. (1979) Receptor affinity and potency effects of omega chain methylation of prostaglandins and prostacyclins. Proc. Int. Prostaglandin Conf., Washington, D.C., p. 4.

Bennett, A. (1974) Prostaglandin antagonists. Adv. Drug. Res. 8, 83-118.

Biggers, J.D., Leonov, B.V., Baskar, J.F. \& Fried, J. (1978) Inhibition of hatching of mouse blastocysts in vitro by prostaglandin antagonists. Biol. Reprod. 19 , 519-533.

Biggers, J.D., Baskar, J.F. \& Torchiana, D.F. (1981) Reduction of fertility of mice by the intrauterine injection of prostaglandin antagonists. J. Reprod. Fert. 63, 365-372.
Cushman, D.w. \& Cheung, H.S. (1976) Effect of substrate concentration on inhibition of prostaglandin synthetase of bull seminal vesicles by anti-inflammatory drugs and fenamic acid analogs. Biochim. Biophys. Acta 424, 449-459.

Dey, S.K., Chein, S.M., Cox, C.L. \& Crist, R.D. (1980) Prostaglandin synthesis in the rabbit blastocyst. Prostaglandins 19, 449-453.

Dickmann, Z. \& Spilman, C.H. (1975) Prostaglandins in rabbit blastocysts. Science, N.Y. 190, 997-998.

Emmens, C.W. (1948) Principles of Biological Assay. Chapman and Hall, London.

Goldstein, L.S., Spindle, A.I. \& Pedersen, R.A. (1975) $\mathrm{X}$-ray sensitivity of the preimplantation mouse embryo in vitro. Radiation Res. 62, 276-287.

Hynie, S., Cepelík, J., Cernohorský, M., Klenerová, V., Skřivanová, J. \& Wenke, M. (1975) 7-Oxa-13prostynoic acid and polyphloretin phosphate as non-specific antagonists of the stimulatory effects of different agents on adenylate cyclase from various tissues. Prostaglandins 10, 971-981.

Kimball, F.A., Lauderdale, J.W., Nelson, N.A. \& Jackson, R.W. (1976) Comparison of luteolytic 
effectiveness of several prostaglandin analogs in heifers and relative binding affinity for bovine luteal prostaglandin binding sites. Prostaglandins 12, 985995.

Langs, D.A., Erman, M. \& DeTitta, G.T. (1977) Conformations of prostaglandin $\mathrm{F}_{2 \alpha}$ and recognition of prostaglandins by their receptors. Science, N.Y. 197, 1003-1005.
Maddox, Y.T., Ramwell, P.W., Shiner, C.S. Corey, EJ. (1978) Amide and 1-amino derivatives of $F$ prostaglandins as prostaglandin antagonists. Nature, Lond. 273, 549-552.

MeDonald-Glbson, R.G., Flack, J.D. \& Ramwell, P.W. (1973) Inhibition of prostaglandin biosynthesis by 7-oxa- and 5-oxa-prostaglandin analogues. Biochem. J. 132, 117-120.

Received 22 December 1980 